\title{
A Novel Fractal Block Coding Method by Using New Shape-based Descriptor
}

\author{
Hsiao-Wen Tin ${ }^{1}$, Shao-Wei Leu ${ }^{1}$, Hiroyuki Sasaki ${ }^{2}$ and Shun-Hsyung Chang ${ }^{3, *}$ \\ ${ }^{1}$ Department of Electrical Engineering, National Taiwan Ocean University, Keelung, Taiwan, R.O.C. \\ ${ }^{2}$ Grassland Management Area, National Institute of Livestock and Grassland Science, Senbonmatsu, Nasushiobara, Japan \\ ${ }^{3}$ Department of Microelectronics Engineering, National Kaohsiung Marine University, Kaohsiung, Taiwan, R.O.C.
}

\begin{abstract}
The basic theory of Jacquin's fractal block coding is to approximate a natural image by its partial subdivided parts, given that the parts resemble the whole at either the same scale or different scales. The fact that self-similarity most commonly exists within an entire natural image leads to the development of the proposed method that uses arc as the fractal descriptor, in which an arc commonly exists within the entire natural image, named as Arc-Descriptor Fractal Coding (ADFC) method. In the ADFC, each range block is approximated by using the selected arc-descriptor from an optimal pool. This paper experimentally demonstrates the ADFC method on Java and the ADFC system is verified with 5 medical images. The experimental results indicate the ADFC method can encode and decode the experimental images effectively. The PSNR of the images after encoding can reach $30 \mathrm{~dB}$ at limited cost while the CR is less than $17 \%$. The ADFC outperforms Jacquin's method under the comparison of the number of search in matching process. We conclude that the ADFC successfully encodes and decodes image in an efficient search during encoding phase without noticeable loss of image quality.
\end{abstract}

Keywords: Descriptor, fractal block coding, image compression, self-similarity

\section{Introduction}

In the '80s, M. Barnsley suggested that Iterated Function Systems consisting of sets of contractive maps with associated probabilities could be used not only to generate fractal sets and measures but also to approximate natural images. After Barnsley, fractal techniques have been applied extensively in several areas of digital image processing, such as image analysis [1] and image coding $[2,3,4]$. Among them, fractal image coding received most attention which fundamental idea based on encoding an image by a contractive transform for which the fixed point is an approximation of the original image.

The block-based fractal image coding method was first introduced by Jacquin [5,6] and Bernesly [7,8]. The fractal block coding method consists of discovering a construction rule to build an approximate image based on the observed tendency for all natural images are rich in affine redundancy. It divides an image into range blocks and domain blocks, and then searches in domain pool for the best matched domain blocks which match the range blocks the most. Fractal block coding achieves excellent compression results because it records only the necessary parameters of transformation in the matching process and correspondent domain blocks during encoding [6,7], [9] , and [10].

During the encoding phase of fractal block coding, the size of domain pool determine how much time the encoding spends in searching the best match for each range block. Therefore, there were many researches on how to obtain the best quality with as little searching as possible. A plenty of studies of some of the researches can be found in $[11,12,13,14]$. One of the simplest ways of decreasing coding time is to decrease the number of domain blocks in order to decrease the number of search [15].

Based on such consideration, this paper proposes a new approach, with the smallest possible pool of self-similar descriptors, to find each range block for its optimal matching self-similarity descriptor according to the best possible accuracy. The proposed method uses a set of elaborative designed arc-descriptors to approximate

\footnotetext{
*Corresponding author e-mail: stephenshchang@mac.com
} 
range blocks. We named the method Arc-Descriptor Fractal Coding (ADFC).

The ADFC approximates the natural image with the elaborative designed arc-descriptor. It is motivated by the observation that natural images are generally quite self-similar, and arc can be found abundantly in the organic shapes of various natural image, in which the organic shapes are shapes with flowing and curving and irregular appearance, and the why the arc-descriptor as a self-similarity can be found within the entire natural image commonly.

The basic concept related to the fractal block coding method of an image is used. The ADFC constructs an image transformation during encoding an image, and then results a fractal code. For an image, the ADFC iteratively generates a set of fractal images to converge to an approximation of the original. In the encoding phase of the ADFC, an image is partitioned into a number of non-overlap range blocks at first. Meanwhile, the ADFC generates a group of arc-descriptors according to the classified image gray values. The number of arc-descriptors is much less than that of domain blocks in conventional fractal coding. Consequently, the ADFC examines how well range blocks are approximated by arc-descriptors, at either the same scale or different scales, and the information of successful approximation is written into the codebook. As a result, the proposed method greatly reduces the number of searches in the matching process.

The ADFC has been implemented in computer software, and 5 medical images were successfully encoded and decoded without noticeable loss of image quality. This paper evaluated the performance of our proposed method by using the image quality after encoding, compression ratio (CR), search-time complexity with peak signal-to-noise ratio (PSNR), the ratio of file sizes, and the searching time in matching process. The experimental results indicate that the proposed method is successful in reducing the number of search in encoding phase with little quality loss and good CR.

The rest of this paper is organized as follows. In the next section, the fractal block coding method is introduced. In section 3, we describe the ADFC. Section 4 illustrates the software implementation and the experiment conducted for the purpose of verifying our method, and analyzes the experimental results which show the consistency with the ADFC. In the final section the concluding remarks are present.

\section{Fractal block coding method}

Mandelbrot based the idea of self-similarity and demonstrated how "fractal" sets could be regarded as limits of iteration involving generators in [16]. In other words, a fractal object is an object which can be assembled by its subdivided parts similar to the whole exactly or statistically. This concept leads to the creation of a class of fractal image coding methods. The first fractal block coding was pioneered by Jacquin [6] and Bernesly [7,8]. The fractal block coding seeks to approximate the image based on the subblocks of that image.

The basic theory of Jacquin's block coding method is described as: let $I$ be a grayscale image. In fractal block coding, image $I$ is partitioned into non-overlapping range blocks $R_{i}, i=1,2, \ldots, N$, so that $I=\cup R_{i}$ and domain blocks $D_{j} \subseteq I, j=1,2, \ldots, M$, where the size of each domain block is larger than that of each range block. To encode an image, each range block will find a domain block most similar to itself from the domain pool, in which the finding is based on minimum mean-squared error criteria. The search is performed with an affine transformation $w_{i}$, such that $w_{i}: D_{j} \rightarrow R_{i}$ where $D_{j}$ is the best matched domain block. A common form of the transformation is shown as:

$$
w_{i}\left[\begin{array}{l}
x \\
y
\end{array}\right]=\left[\begin{array}{ll}
a_{i} & b_{i} \\
c_{i} & d_{i}
\end{array}\right]\left[\begin{array}{l}
x \\
y
\end{array}\right]+\left[\begin{array}{l}
e_{i} \\
f_{i}
\end{array}\right]
$$

Put a constrain to the transformation $w_{i}$ for contraction so that for any two points $p_{1}$ and $p_{2}$, the distance $d()$ between two points should fulfill the inequality:

$$
d\left(w_{i}\left(p_{1}\right), w i\left(p_{2}\right)\right)<\alpha d\left(p_{1}, p_{2}\right)
$$

where $\alpha$ is a coefficient and $\alpha<1$. To encode an image would start from performing the transformation $w_{i}$ on domain block $D_{j}$ for a range block $R_{i}$, based on equations (1) and (2) it derives the following:

$$
w_{i}\left(D_{j}\right)=\alpha D_{j}+c_{0}
$$

where $c_{0}$ is a coefficient. Theoretically, the union of the affine transformations $\tau$ for all range blocks will form the affine transformation for the whole image as expressed in equation (4) $[8,17]$.

$$
\tau=\bigcup_{i=1}^{N} w_{i}
$$

The encoding method would seek for a transformation of domain block to the best approximation of a selected range block. To determine the $\tau$ and $c_{0}$ for exactly transformation on each domain block, it should find the minimum distance between range block and domain block:

$$
\min \Sigma_{n, m}\left(R_{i}\right)_{n, m}-\left(\tau\left(D_{j}\right)\right)_{n, m}
$$

where $n$ and $m$ are the sizes of blocks, usually are set to 2 or 4 . The encoding method uses the following distance equation to compare the range block with domain block for determining the best matching:

$$
d\left(\tau\left(D_{j}\right), R_{i}\right)=\Sigma\left(\tau\left(D_{j}\right)-R_{i}\right)^{2}
$$


Image encoding is achieved by recording the $\tau$, the minimal distance, and the respect $D_{j}$. Fractal codes recorded in the codebook can later be used in approximating the image.

To decode the image, the coding method would perform the transformation iteratively on some initial image $\Omega_{\text {init }}$ stored in the code book until the encoded image is restored. The decoding process for $K$ th iteration is described as:

$$
\Omega_{k}=\tau(\Omega) \psi(\Omega)
$$

where $\tau$ is the transformation and $\psi$ is the ensemble function to assemble the transformations.

\section{The Proposed arc-descriptor fractal coding method}

As mentioned above, the Jacquin's method approximates image subblocks by a number of other blocks. The main difference between the ADFC and the Jacquin's method is that the ADFC uses a set of arc-descriptors to approximate the image subblocks. A block diagram in Fig. 1 explains the process of the ADFC. In the encoding phase, an image is partitioned into a collection of range blocks. At the same time, a set of arc-descriptors is created. For each range block, the search in the arc-descriptor pool is performed with affine transformations to obtain the best matched arc-descriptor, in which the finding is based on minimum mean-squared error criterion. Image encoding is achieved by storing the transformations and arc-descriptors in the codebook. In the decoding phase, image decoding is performed by approximating the range blocks iteratively by performing the affine transformations with the recorded arc-descriptors.

To encode a gray image $I$, the ADFC firstly divides the image into non-overlapping square blocks which sizes are $I \times I$ as the range blocks. Then, the ADFC for each gray values exhibited in image creates arc-descriptors respectively which sizes are larger than that of range block. For each range block $R_{i}$, the ADFC applies the affine transformation $w_{i}$ to arc-descriptor $A_{j}$, and compares the transformation result to the range block. The arc-descriptor with the minimal distortion is said to be the best matched arc-descriptor for the range block, just similar to equation (5):

$$
\min \Sigma_{n, m}\left(R_{i}\right)_{n, m}-\left(\tau\left(A_{j}\right)\right)_{n, m}
$$

The minimal distance is defined as following equation similar to equation (6):

$$
d\left(\tau\left(A_{j}\right), R_{i}\right)=\Sigma\left(\tau\left(A_{j}\right)-R_{i}\right)^{2}
$$

The ADFC records the transformation $\tau$, the minimal distance $d()$, and the respect $A_{j}$ in fractal codebook. The

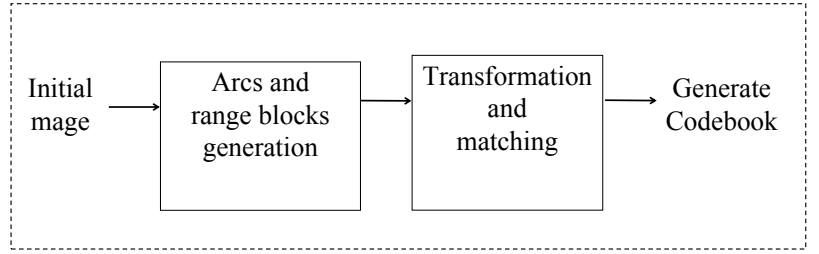

Encoding Phase

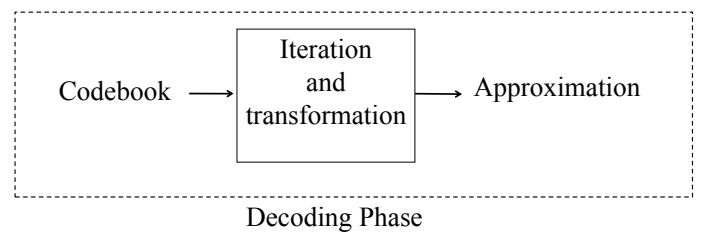

Fig. 1: Arc-descriptor fractal coding schema.

decode process is as the same as the fractal block coding method.

In the next, this paper demonstrates how to create an arc-descriptor. In general, an arc is any smooth curve connecting two points. Let angle be $\theta$, and radius be $R$. Let $\left(x_{0}, y_{0}\right)$ be the center point of arc. The arc diagram is illustrated as Fig. 2.

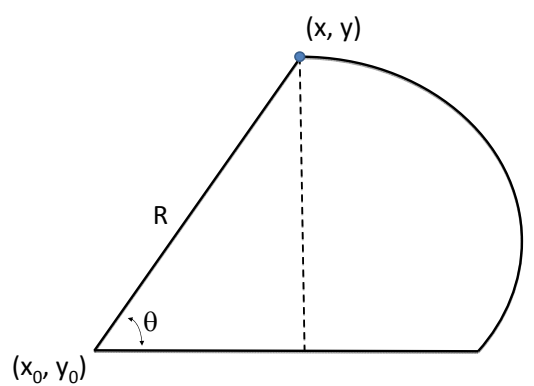

Fig. 2: Arc expression by angle and radius.

For point $(x, y)$ on an arc, a common equation for the arc in the angle $\theta$ ' is expressed as,

$$
\begin{aligned}
& x=R \times \cos \theta^{\prime}+x_{0} \\
& y=R \times \sin \theta^{\prime}+y_{0}
\end{aligned}
$$

As shown in Figure 2, the arc center point, angle $\theta$, radius $R$, and start point $S$ are known, then connecting point on arc can be obtained alongside the arc angle, also the end point $E$ can be calculated by equation (10). Therefore, the arc $\overparen{S E}$ is built by connecting the start point and end point.

Theoretically the number of arc-descriptors is chosen as many as the number of gray values exhibited in image. 
However, the operation is complicate and difficult, and this paper expects the ADFC achieves the effective approximation of range blocks with as less as possible of arc-descriptors. Therefore, this paper partitions the gray values into several classes, and takes the lowest gray value in each class as the index gray value for that class. The ADFC creates the arc-descriptor for each class, and assigns the gray value as the same as the respected class. The selection of an optimal number of classes depends on experimental experience.

Here, assume $\mathrm{cls}$ arc-descriptors to form the optimal pool make the best possible accuracy. A bottom threshold $r$ used in the creation of arc-descriptors based on $\mathrm{cls}$ is obtained by using floor function as expressed in equation (11).

$$
r=\left\lfloor\frac{G_{\max }+1-G_{\min }}{c l s}\right\rfloor
$$

where $G_{\max }$ and $G_{\min }$ represent the maximum and minimum gray values respectively in image.

Next, the ADFC creates a series of thresholds based on $r$, and consequently lays the gray values of the image within the thresholds to form a set of gray value classes. The arc-descriptor pool comprises arcs each of which is built and designated a gray value according to the gray value classes. For instance, class 1 contains the gray values $G_{\min }$ to $(r \times 1)-1$, class 2 contains the pixels with gray value $(r \times 1)$ to $(r \times 2)-1$, class $i$ contains the pixels with gray value $(r \times(r-1))$ to $(r \times i)-1$, until all of the pixels are assigned to their designated classes. The minimal gray value in each class is assigned to be the gray value of that class. Consequently, we create $\mathrm{cls}$ arc-descriptors for each class and assign arc-descriptors the gray values as the same as the associated classes.

\section{Experiment and Analysis}

\subsection{System Algorithm and Parameters}

This paper experimentally demonstrates the ADFC on Java and uses in implementation 5 EM images as the experimental data. The flow of the ADFC encoding algorithm is described as follows.

step 1:Read the grayscale medical image..

step 2:Segment the image using a fixed block size into range blocks. A $2 \times 2$ block size is selected as the same as that used in Jacquin [6] and Davis [3].

step 3:Select a number for coefficient cls. Obtain a gray value series based on bottom threshold calculated by using equation (11). For each gray value in that series, create an arc-descriptor with the same gray value using a block size twice the range size, which is $4 \times 4$ as the same as the size of domain block used in Jacquin [6] and Davis [3]. By averaging four pixels each shrinks the arc-descriptor to match the size of the range blocks. This step produces the codebook of arc-descriptors $A_{j}$. step 4:For each range block $R_{i}$ an optimal approximation $R_{i}=\alpha A_{j}+c_{0}$ is determined as follows:

1.Compute the distances between range block $R_{i}$ and all of the arc-descriptors $d\left(\tau\left(A_{j}\right), R_{i}\right)=\Sigma\left(\tau\left(A_{j}\right)-\right.$ $\left.R_{i}\right)^{2}$.

2.The arc-descriptor said $A_{j}$ with the minimal distance to range block $R_{i}$, $\min \Sigma_{n, m}\left(R_{i}\right)_{n, m}-\left(\tau\left(A_{j}\right)\right)_{n, m}$, is the best matched arc-descriptor.

3. Record the code for the range block $R_{i}$.

step 5:After all of the range blocks are processed by step

4 , the encoding is completed.

The decoding of the ADFC is as the same as the conventional fractal block decoding. Start from an initial image. Then perform the transformation iteratively on the initial image based on the recorded code.

This paper uses 5 EM images for experimentation which are from the U.S. Centers for Disease Control and Prevention. All of the images are converted into $300 \times 300$ pixel bmp images as illustrated in Fig. 3 .

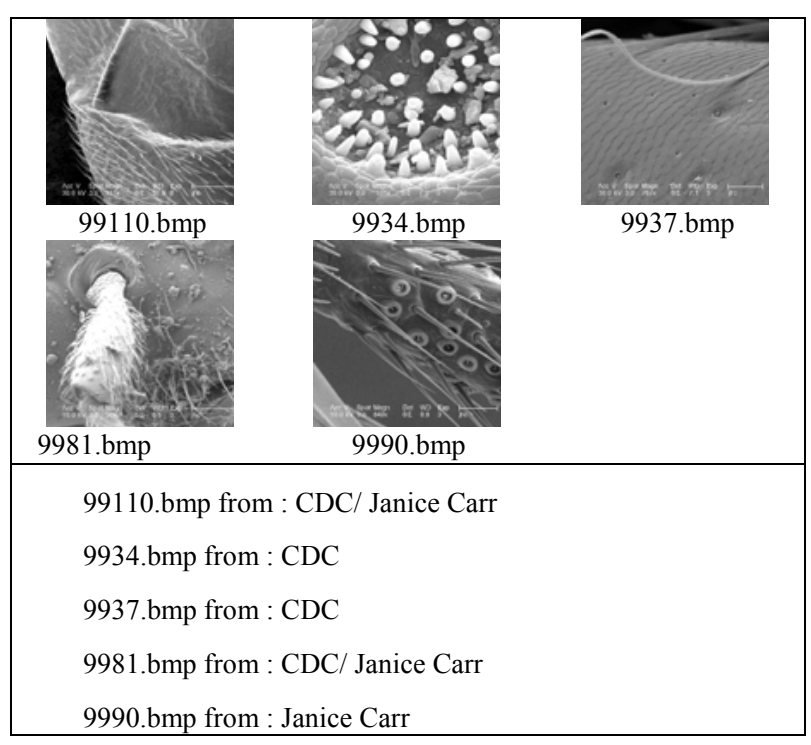

Fig. 3: Original EM images used in proposed method.

The ADFC is tested with various performance evaluation parameters including PSNR and CR. The PSNR is described as follows. Let $A(x, y)$ be the gray value expression of the original image $A$. Let $B(x, y)$ be the gray value expression of the image $B$ after encoding. The images are of the same size $N \times M$. Let $I_{\max }$ be the maximum gray value. The mean square error (MSE) and 
the PSNR is defined in equation (12).

$$
\begin{gathered}
M S E=\frac{\sum_{x=0}^{N-1} \sum_{y=0}^{M-1}[B(x, y)-A(x, y)]^{2}}{N \times M} \\
P S N R=10 \log \left(\frac{I_{\max } \times I_{\max }}{M S E}\right) d B
\end{gathered}
$$

The CR is defined as the ratio of the number of bits represents the size of original image to the number of bits represents the size compressed image. CR show that how much times the image has been compressed.

$$
C R=\frac{n_{\text {original }}}{n_{\text {compressed }}}
$$

where $n_{\text {original }}$ and $n_{\text {compressed }}$ are the numbers of bits required to represent the original and compressed image.

\subsection{Search Time Complexity Analyses}

Assume the time spent in matching process for an image with a number of range blocks in Jacquin's method, and the ADFC are both with brute-force search scheme, and do not count on the affine transformation. The time spent in Jacquin's method is the product of the number of range block and the number of domain block. On the other hand, the time spent in the ADFC is the product of the number of range block and the number of arc-descriptor.

Let $N_{r}$ be the number of range blocks. In Jacquin's method, for each range block, the best matched domain block in domain pool with $N_{d}$ domain blocks is searched. The time complexity for every range block searching the domain blocks is $O\left(N_{r} \times N_{d}\right)$.

In the ADFC, let $c l s$ be set to the same as the largest possible gray value, 256, for obtaining the best possible accuracy; therefore, 256 arc-descriptors are created. For each range block, the best matched one in the 256 arc-descriptors is searched. The time complexity for every range block searching the arc-descriptors is $O\left(N_{r} \times 256\right)$.

When the number of domain blocks in Jacquin's fractal block coding method is larger than 256, the number of search by using Jacquin's method is larger than that by using the ADFC. In such a case, the time complexity of the ADFC is better than that of Jacquin's method.

\subsection{Experimental Results and Analyses}

Although applying 256 arc-descriptors created for all possible gray values in image would lead the ADFC system to an excellent result, it degrades the performance due to redundant arc-descriptors. To obtain the best possible number of arc-descriptors, this paper has compared various decoding results of the ADFC obtained by using various numbers, from 2 to 10 , of arc-descriptors. Fig. 4 demonstrated the 9990.bmp after the ADFC encoding with various numbers of arc-descriptors.
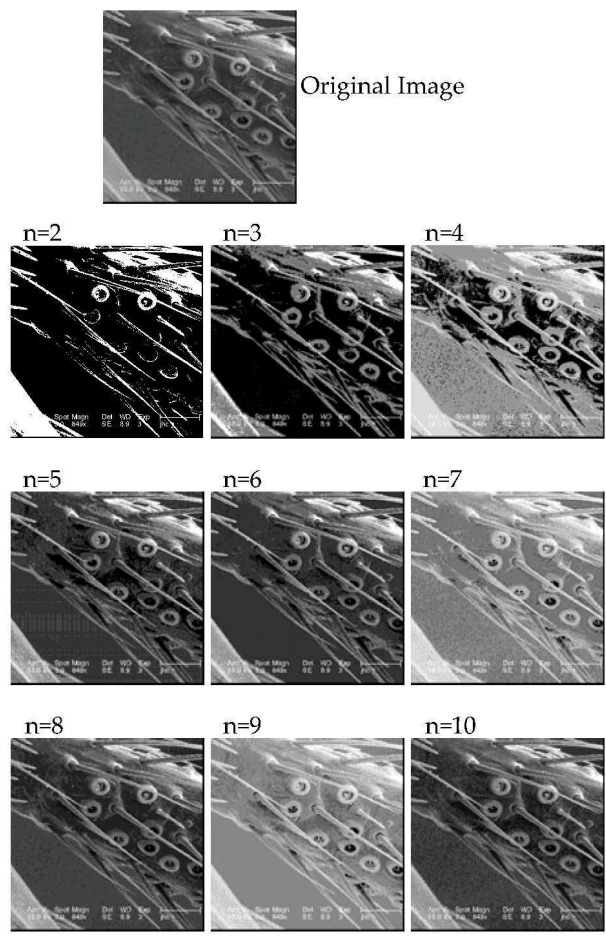

Fig. 4: Decoding images which were encoded with $\mathrm{n}$ arcdescriptors of size $4 \times 4$. The PSNR increases as the number of arc-descriptors applied increases.

To determine the optimal number cls of arc-descriptors in pool, this paper compares the parameter $c l s$ with respect to PSNR. The experimental PSNR results of various EM images obtained by using various numbers of arc-descriptors are listed in Table 1. Those images have been carried out in the scenarios discussed as the number of arc-descriptors against the image quality after encoding. The PSNR results indicates the situation while increasing the number of arc-descriptors, we obtain better quality of decoded result.

Table 1 lists the results of CR. It indicates the CR value increases as the number of arc-descriptors increases. In this experiment, the $\mathrm{CR}$ values in 10 arc-descriptors which results acceptable PSNR of all images are between $9.54 \%$ and $16.5 \%$.

This paper also processed the 5 EM images with conventional fractal coding method. The resulted PSNR and CR are listed in Table 1. Although the CR values in conventional fractal coding are quite uniform, the PSNR values are not good enough. Apparently the ADFC is competitive to conventional fractal coding. 
Table 1: PSNR and CR of images after the ADFC encoding and conventional fractal block encoding

\begin{tabular}{|l|l|l|l|}
\hline \multicolumn{4}{|c|}{ Fractal Block Coding } \\
\hline & Image & PSNR(dB) & CR(\%) \\
\hline & 9910 & 24.446 & 10.62 \\
& 9934 & 27.215 & 11.01 \\
& 9937 & 29.4 & 9.77 \\
& 9981 & 29.4 & 10.70 \\
& 9990 & 26.238 & 10.46 \\
\hline \hline \multicolumn{4}{|c|}{ ADFC } \\
\hline & Image & PSNR(dB) & CR(\%) \\
\hline \multirow{5}{*}{5 arc-descriptors } & 9910 & 32.665 & 10.58 \\
& 9934 & 24.633 & 9.73 \\
& 9937 & 34.778 & 5.86 \\
\hline \multirow{5}{*}{10 arc-descriptors } & 9981 & 24.882 & 10.66 \\
& 9990 & 33.646 & 9.15 \\
\hline \multirow{5}{*}{ 15 arc-descriptors } & 9910 & 39.13 & 16.45 \\
& 9934 & 30.816 & 16.07 \\
& 9937 & 42.382 & 9.54 \\
& 9981 & 31.547 & 16.50 \\
& 9990 & 40.721 & 14.62 \\
\hline & 9910 & 44.706 & 20.23 \\
& 9934 & 36.674 & 18.17 \\
& 9981 & 46.819 & 12.91 \\
& 9990 & 36.923 & 19.65 \\
& 9910 & 42.688 & 17.04 \\
\hline & 9934 & 33.933 & 23.36 \\
& 9937 & 45.228 & 21.60 \\
& 9981 & 34.553 & 22.60 \\
& 9990 & 43.644 & 20.00 \\
\hline
\end{tabular}

The experimental results indicate that the arc-descriptor is well suitable as the self-similarity of fractal coding. This actually substantiates why an arc can be found abundantly in the organic shapes of various natural images.

\section{Conclusion}

This paper designed the ADFC to encode the image at the best possible accuracy with the smallest possible arc-descriptor pool and its experiment examines how well range blocks are approximated by arc-descriptors, at either the same scale or different scales. The following points highlight the main contributions of this paper.

1.This paper proposes a novel method to successfully encoded and decoded without noticeable loss of image quality, of which the PSNR values are acceptable while $\mathrm{CR}$ values are still low.

2.The ADFC consists of a set of arc-descriptors to obtain the best possible accuracy with the optimal arc-descriptor pool. The ADFC outperforms to conventional fractal block coding in search time complexity.
The high PSNR and low CR of 5 medical images after the ADFC encoding indicate that the ADFC can effectively approximate the natural image with arc-descriptor, which shape is arc, of course, that can be found abundantly in many natural images. This actually explains why the arc-descriptor commonly exists within the entire natural image. The experimental results also indicate that the size of the optimal arc-descriptor pool is decreased and the time complexity can save very significantly compared to Jaquin's method.

This paper has demonstrated that arc-descriptor is suitable to be self-similarity descriptor to encode various natural images. The PSNR and CRs are varied by the number of arc-descriptors. The ADFC does achieve the goal of improving the efficiency while preserving the desired image quality. The result has encouraged us to proceed to remote sensing image study.

\section{Acknowledgement}

This work was supported by the National Science Council of Taiwan, R.O.C. under contract NSC99-2923-E-022-001-MY3.

The authors are grateful to the anonymous referee for a careful checking of the details and for helpful comments that improved this paper.

\section{References}

[1] A. N. Esgiar, R. N. G. Naguib, B. S. Sharif, M. K. Bennett, and A. Murray, Fractal analysis in the detection of colonic cancer images, IEEE Trans. on Information Technology in Biomedicine, 6, 5458 (2002).

[2] L. Anson, Fractal image compression, BYTE magazine, (1993).

[3] G. M. Davis, A Wavelet-Based Analysis of Fractal Image Compression, IEEE Trans. Image Processing, 7, 141-154 (1998).

[4] M. M. Chelehgahi, K. Jaferzadeh, M. D. Nia, A high speed intelligent classification algorithm for fractal image compression using DCT coefficients, Proc. 3rd IEEE International Conf. on Communication Software and Networks (ICCSN), 156-159 (2011).

[5] A. E. Jacquin, Fractal image coding: a review, Proceedings of the IEEE, 81, 1451-1465 (1993).

[6] A. E. Jacquin, Image Coding Based on a Fractal Theory of Iterated Contractive Image Transformations, IEEE Trans. Image Processing, 18-30 (1992).

[7] M. F. Barnsley, S. Demko, Iterated function systems and global construction of fractals, Proceedings of the Royal Society of London, 243-275 (1985).

[8] M. F. Barnsley, L. P. Hurd, Fractal Image Compression. AK Peters, Ltd. Wellesley, Massachusetts, (1993).

[9] J. Hutchinson, Fractals and Self-Similarity, Indiana University Journal of Mathematics, 30, 713-747 (1981).

[10] N. Lu, Fractal image, Academic Press. Boston, (1996). 
[11] T. ás Kovács,, A fast classification based method for fractal image encoding. Image and Vision Computing, 26, 11291136 (2008).

[12] D. J. Duh, J. H. Jeng, S. Y. Chen, DCT based simple classification scheme for fractal image compression, Image and Vision Computing, 23, 1115-1121 (2005).

[13] J. C. C. Tseng, and J. G. Hsieh, Study on Huber Fractal Image Compression, IEEE Trans. on Image Processing, 9951003 (2009).

[14] H. W. Tin, S. W. Leu, S. H. Chang, An Efficient Approach to Speed up the Search Process during Fractal Matching Process, Proc. 12th Computer Engineering and Applications, 110-115 (2012).

[15] B. Wohlberg, G. Jager, A review of the fractal image coding literature, IEEE Trans. on Image Processing, 8, 1716-1729 (1999).

[16] B. B. Mandelbrot, Fractal Geometry of Nature, San Francisco. CA:Freeman, (1982).

[17] E. W. Jacobs, Y. Fisher and R. D. Boss, Image compression: A study of the iterated transform method, Signal Processing, 29, 251-263 (1992).

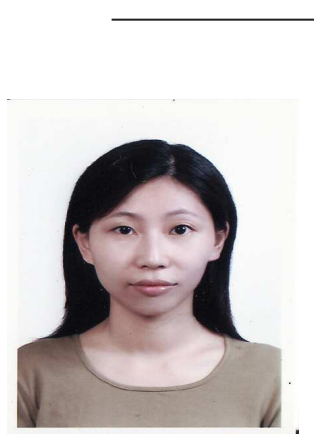

Hsaio-wen Tin received the M.S. degree in Applied Mathematics from National Chung Hsing University, Taichung, Taiwan, in 2001, and the Ph.D. degree from the Department of Electrical Engineering, National Taiwan Ocean University, Keelung, Taiwan, in 2013. Her major field of study is signal processing and image processing. Since 2008, she has published her researches to 2008 International Computer Symposium, Taiwan, International Computer Symposium 2010 (ICS 2010), Taiwan and Computer Engineering and Applications (CEA'12), Cambridge, MA, USA.

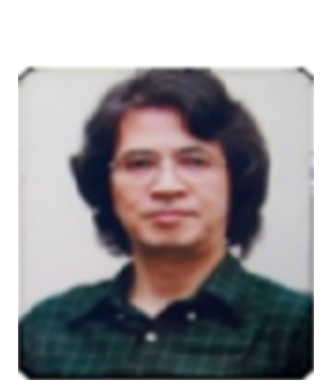

\section{Shao-Wei}

Leu received the B.S. degree in Biomedical Engineering from Chung-Yuan Christian University, Chung-Li, Taiwan, in 1979, the M.S. degree in Computer Engineering from Clemson University, Clemson, South Carolina, in 1985, and the Ph.D. degree from Florida Atlantic University, Boca Raton, Florida, in 1990. He has been an associate professor in the Department of Electrical Engineering at National Taiwan Ocean University, Keelung, Taiwan, since 1990. His research interests include VLSI system design and verification, parallel processing, and network computing.

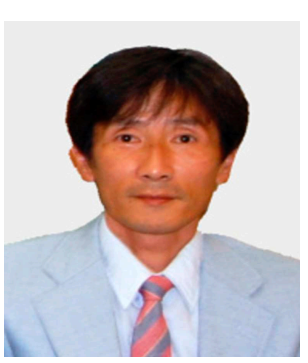

Hiroyuki Sasaki received the M.Ag. degree in "Planting distribution of evergreen broad-leaved trees" from Tokyo University, Japan, in 1988, and has been researcher in the National Institute of Livestock and Grassland Science in Japan. In 1999, he received Research Encouragement Prize of the Japanese society of grassland science in "Computer systems to support land use planning in pasture", and received the Ph.D. degree in "Greenhouse effect on the productivity of forage grass" from Kyushu University, Fukuoka, Japan, in 2004. He received the Prize of Hokkaido society of grassland science in 2007 about "Development of decision support system for application of manure and fertilizer to grassland and forage corn field based on nutrient recycling". His software named "Fractal analysis system" is used all over the world.

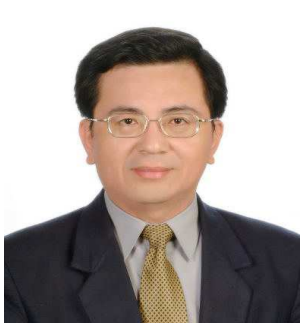

Shun-Hsyung Chang received the Ph.D. Degree in electrical engineering from the National Sun Yat Sen University, Taiwan, in 1990. Afterwards, he served as an associate professor and has been a professor since 1997 in the Department of Electrical Engineering at National Taiwan Ocean University where he was the chairman 1998-2003. Since August 2003, he also has been serving as the Vice President of the National Kaohsiung Marine University, Taiwan, and a professor in the Department of Microelectronic Engineering at this university. He has published over 230 technical papers. His research interests include underwater signal processing, electrical engineering, and communication engineering. His outstanding performance in research brought him great honor. In 1984, he was granted "The Creative Youth" prize by the Ministry of Education. He received the Long- Term Paper Award, organized by Acer Incorporated, in 1994, 1996 and 1998. He jointly with his graduate students won the Third Prize of Graduate Team in TI-Taiwan 1994 DSP Design Championship. $\mathrm{He}$ contributes greatly in editorship. He was the editor-in-chief of the Journal of Marine Science and Technology 1998-2001, of which he is an editor at present. He is currently an editor-in-chief of the Journal of Ocean and Undersea Technology 2008-2011. 